\title{
Supporting Information: Design of Non-Ideal Eutectic Mixtures Based on Correlations with Molecular Properties
}

Laura J.B.M. Kollau, ${ }^{\dagger, \ddagger}, \mathbb{\Phi}$ Remco Tuinier, ${ }^{*, \dagger, \ddagger}$ Job Verhaak, ${ }^{\dagger}$ Jaap den Doelder, ${ }^{\dagger, \S}$ Ivo A.W. Filot, $\|, \ddagger$ and Mark Vis ${ }^{\dagger, \ddagger}$

$\dagger$ Laboratory of Physical Chemistry, Department of Chemical Engineering and Chemistry, Eindhoven University of Technology, P.O. Box 513, 5600 MB Eindhoven, The Netherlands $\ddagger$ Institute for Complex Molecular Systems, Eindhoven University of Technology, P.O. Box 513, 5600 MB Eindhoven, The Netherlands

\Laboratoire de Chimie, École Normale Supérieure de Lyon \& CNRS, 46 Allée d'Italie, 69007 Lyon, France

§Packaging and Specialty Plastics REDD, Dow Benelux BV, P.O. Box 48, 4530AA Terneuzen, The Netherlands

|l Laboratory of Inorganic Materials and Catalysis, Department of Chemical Engineering and Chemistry, Eindhoven University of Technology, The Netherlands

E-mail: r.tuinier@tue.nl 


\section{Supporting information}

Final model equation:

$$
\begin{aligned}
\chi=C_{0} & +C_{D_{1}} \cdot D_{1}+C_{D_{5}} \cdot D_{5}+C_{A_{2}} \cdot A_{2}+C_{A_{4}} \cdot A_{4}+C_{\mathrm{NL} 1} \cdot\left(D_{1}-\left\langle D_{1}\right\rangle\right)^{2} \\
& +C_{\mathrm{NL} 2} \cdot\left[\left(A_{2}-\left\langle A_{2}\right\rangle\right) \cdot\left(D_{5}-\left\langle D_{5}\right\rangle\right)\right]+C_{\mathrm{NL} 3} \cdot\left[\left(D_{5}-\left\langle D_{5}\right\rangle\right) \cdot\left(A_{4}-\left\langle A_{4}\right\rangle\right)\right]
\end{aligned}
$$

Table S1: Parameters for eq. (S1).

\begin{tabular}{cc}
\hline Constant & Value \\
\hline$C_{0}$ & 232.41 \\
$C_{D_{1}}$ & 1.9866 \\
$C_{D_{5}}$ & -1.3315 \\
$C_{A_{2}}$ & -60.439 \\
$C_{A_{4}}$ & 0.8054 \\
$C_{\mathrm{NL}_{1}}$ & -1.2786 \\
$C_{\mathrm{NL}_{2}}$ & 113.87 \\
$C_{\mathrm{NL}_{3}}$ & -2.2432 \\
\hline
\end{tabular}


Table S2: Predicted and measured eutectic fusion properties of the mixtures.

\begin{tabular}{|c|c|c|c|c|c|c|}
\hline Donor & Acceptor & $\chi$ & $\sigma_{\chi}$ & $T_{\mathrm{e}}(\mathrm{K})$ & $\chi_{\text {measured }}$ & $T_{\mathrm{e}, \text { measured }}(\mathrm{K})$ \\
\hline Succinic Acid & $\mathrm{Et}_{4} \mathrm{NBr}$ & -8.24 & 0.75 & $333.41-348.19$ & -7.92 & 352.60 \\
\hline Glutaric Acid & $\mathrm{Et}_{4} \mathrm{NBr}$ & -5.63 & 0.58 & $350.28-362.17$ & -5.44 & 303.30 \\
\hline Adipic Acid & $\mathrm{Et}_{4} \mathrm{NBr}$ & -5.44 & 0.49 & 299.92-309.92 & -5.53 & 352.50 \\
\hline Pimelic Acid & $\mathrm{Et}_{4} \mathrm{NBr}$ & -4.58 & 0.52 & $321.45-332.07$ & -4.29 & 323.50 \\
\hline Suberic Acid & $\mathrm{Et}_{4} \mathrm{NBr}$ & -4.07 & 0.09 & $350.64-352.34$ & & \\
\hline Erythritol & $\mathrm{Et}_{4} \mathrm{NBr}$ & -4.19 & 0.23 & $352.93-358.96$ & -4.79 & 346.10 \\
\hline Xylitol & $\mathrm{Et}_{4} \mathrm{NBr}$ & -1.89 & 0.37 & $312.28-321.69$ & & \\
\hline Sorbitol & $\mathrm{Et}_{4} \mathrm{NBr}$ & -3.07 & 0.38 & $326.60-337.34$ & & \\
\hline Succinic Acid & $\mathrm{Pr}_{4} \mathrm{NBr}$ & -2.92 & 0.42 & $315.58-324.03$ & & \\
\hline Glutaric Acid & $\mathrm{Pr}_{4} \mathrm{NBr}$ & -2.58 & 0.36 & $310.14-319.69$ & & \\
\hline Adipic Acid & $\mathrm{Pr}_{4} \mathrm{NBr}$ & -2.90 & 0.59 & $345.45-352.46$ & -3.67 & 344.90 \\
\hline Pimelic Acid & $\mathrm{Pr}_{4} \mathrm{NBr}$ & -2.86 & 0.52 & $311.74-318.34$ & -2.26 & 321.00 \\
\hline Suberic Acid & $\mathrm{Pr}_{4} \mathrm{NBr}$ & -3.06 & 0.02 & $343.06-343.33$ & & \\
\hline Erythritol & $\mathrm{Pr}_{4} \mathrm{NBr}$ & -0.84 & 0.37 & $325.16-329.43$ & & \\
\hline Xylitol & $\mathrm{Pr}_{4} \mathrm{NBr}$ & -1.16 & 0.59 & $317.83-326.10$ & & \\
\hline Sorbitol & $\mathrm{Pr}_{4} \mathrm{NBr}$ & -1.04 & 0.24 & $326.95-331.39$ & -0.64 & 336.30 \\
\hline Succinic Acid & $\mathrm{Bu}_{4} \mathrm{NBr}$ & -1.27 & 0.58 & $307.77-320.43$ & -1.30 & 355.80 \\
\hline Glutaric Acid & $\mathrm{Bu}_{4} \mathrm{NBr}$ & -1.98 & 0.35 & $360.86-353.16$ & & \\
\hline Adipic Acid & $\mathrm{Bu}_{4} \mathrm{NBr}$ & -2.54 & 0.24 & $372.39-365.45$ & & \\
\hline Pimelic Acid & $\mathrm{Bu}_{4} \mathrm{NBr}$ & -2.89 & 0.30 & $365.40-362.22$ & & \\
\hline Suberic Acid & $\mathrm{Bu}_{4} \mathrm{NBr}$ & -3.42 & 0.02 & $307.38-308.05$ & -3.15 & 315.90 \\
\hline Erythritol & $\mathrm{Bu}_{4} \mathrm{NBr}$ & -0.11 & 0.02 & 298.32-297.87 & & \\
\hline Xylitol & $\mathrm{Bu}_{4} \mathrm{NBr}$ & -1.64 & 0.43 & $308.30-314.05$ & -2.24 & 313.20 \\
\hline Sorbitol & $\mathrm{Bu}_{4} \mathrm{NBr}$ & -0.92 & 0.02 & $298.22-298.61$ & -1.03 & 312.50 \\
\hline Succinic Acid & $\mathrm{Pe}_{4} \mathrm{NBr}$ & -3.92 & 0.20 & $333.07-337.38$ & -4.01 & 349.30 \\
\hline Glutaric Acid & $\mathrm{Pe}_{4} \mathrm{NBr}$ & -4.26 & 0.54 & $335.52-341.97$ & & \\
\hline Adipic Acid & $\mathrm{Pe}_{4} \mathrm{NBr}$ & -4.74 & 0.36 & $295.71-303.64$ & & \\
\hline Pimelic Acid & $\mathrm{Pe}_{4} \mathrm{NBr}$ & -4.96 & 0.39 & 286.33-293.86 & -5.95 & 309.60 \\
\hline Suberic Acid & $\mathrm{Pe}_{4} \mathrm{NBr}$ & -5.37 & 0.55 & $351.11-363.81$ & & \\
\hline Erythritol & $\mathrm{Pe}_{4} \mathrm{NBr}$ & -2.44 & 0.57 & $332.22-347.77$ & -1.90 & 346.40 \\
\hline Xylitol & $\mathrm{Pe}_{4} \mathrm{NBr}$ & -3.55 & 0.59 & $323.49-330.88$ & -2.77 & 332.40 \\
\hline Sorbitol & $\mathrm{Pe}_{4} \mathrm{NBr}$ & -3.04 & 0.61 & $293.10-304.29$ & -3.43 & 319.70 \\
\hline Succinic Acid & $\mathrm{He}_{4} \mathrm{NBr}$ & -0.96 & 0.44 & $367.51-357.14$ & & \\
\hline Glutaric Acid & $\mathrm{He}_{4} \mathrm{NBr}$ & -1.50 & 0.49 & $354.03-343.01$ & & \\
\hline Adipic Acid & $\mathrm{He}_{4} \mathrm{NBr}$ & -2.45 & 0.45 & $351.63-342.65$ & -2.16 & 322.00 \\
\hline Pimelic Acid & $\mathrm{He}_{4} \mathrm{NBr}$ & -3.45 & 0.32 & $351.16-355.99$ & & \\
\hline Suberic Acid & $\mathrm{He}_{4} \mathrm{NBr}$ & -4.53 & 0.40 & $337.32-345.92$ & & \\
\hline Erythritol & $\mathrm{He}_{4} \mathrm{NBr}$ & 0.60 & 0.44 & $299.18-306.74$ & & \\
\hline Xylitol & $\mathrm{He}_{4} \mathrm{NBr}$ & -2.97 & 0.60 & $326.77-339.67$ & & \\
\hline Sorbitol & $\mathrm{He}_{4} \mathrm{NBr}$ & -1.23 & 0.39 & $300.98-310.14$ & & \\
\hline
\end{tabular}


Table S3: Physical properties of the individual components related to the molecular structures. The molecular mass $M$, density of the solid crystalline components $\rho$, the molecular volume $V_{\mathrm{m}}$, Number $(\#)$ of: $=\mathrm{CO}$ moieties, $-\mathrm{COH}$ moieties, and $-\mathrm{CH}_{2}$ groups. Properties of the individual components related to the acidity of the hydrogen bonding moieties. The solubility in $\mathrm{H}_{2} \mathrm{O}$ at $T=298 \mathrm{~K}$ in $\mathrm{mol} \%$, the proton affinity $\mathrm{PA}, \mathrm{p} K_{\mathrm{a}}$ values, and the molecular dipole moments in $\mathrm{D}$.

\begin{tabular}{ccccc}
\hline Component & $\begin{array}{c}\# \\
\end{array}$ & $\begin{array}{c}\# \\
-\mathrm{COH}\end{array}$ & $\begin{array}{c}\mathrm{H}_{2} \mathrm{O} \text { solubility } \\
{[\mathrm{mol} \% \text { at } 298 \mathrm{~K}]}\end{array}$ & $\mathrm{p} K_{\mathrm{a}}$ \\
\hline Succinic acid & 2 & 2 & $8.85 \cdot 10^{-3}[1]$ & $4.16^{[2]}$ \\
Glutaric acid & 2 & 2 & $8.73 \cdot 10^{-2}[1]$ & $4.32^{[2]}$ \\
Adipic acid & 2 & 2 & $1.78 \cdot 10^{-3}[1]$ & $4.43^{[2]}$ \\
Pimelic acid & 2 & 2 & $5.62 \cdot 10^{-3}[1]$ & $4.47^{[2]}$ \\
Suberic acid & 2 & 2 & $1.65 \cdot 10^{-4}[1]$ & $4.51^{[3]}$ \\
Erythritol & 0 & 4 & $1.48 \cdot 10^{-2}[4]$ & $13.04[1]$ \\
Xylitol & 0 & 5 & $2.37 \cdot 10^{-1}[5]$ & $12.70^{[1]}$ \\
Sorbitol & 0 & 6 & $2.32 \cdot 10^{-1}[6]$ & $12.59[1]$ \\
Et ${ }_{4} \mathrm{NBr}$ & - & - & $2.70 \cdot 10^{-3}[7]$ & - \\
$\mathrm{Pr}_{4} \mathrm{NBr}$ & - & - & $1.93 \cdot 10^{-1}[2]$ & - \\
$\mathrm{Bu}_{4} \mathrm{NBr}$ & - & - & $3.87 \cdot 10^{-1}[2]$ & - \\
$\mathrm{Pe}_{4} \mathrm{NBr}$ & - & - & $4.18 \cdot 10^{-3}[2]$ & - \\
$\mathrm{He}_{4} \mathrm{NBr}$ & - & - & $3.42 \cdot 10^{-4}[2]$ & - \\
\hline
\end{tabular}

Table S4: Hansen solubility parameters of the components used. $\delta \mathrm{D}$ is the dispersion Hansen solubility parameter, $\delta \mathrm{P}$ is the polar Hansen solubility parameter, and $\delta \mathrm{H}$ is the hydrogen bonding Hansen solubility parameter. Values are calculated using a group contribution method. ${ }^{8,9}$

\begin{tabular}{cccl}
\hline Component & $\delta \mathrm{D}$ & $\delta \mathrm{P}$ & $\delta \mathrm{H}$ \\
\hline Succinic acid & 16.7 & 8.6 & 14.6 \\
Glutaric acid & 16.7 & 8.3 & 14.2 \\
Adipic acid & 16.6 & 8 & 13.8 \\
Pimelic acid & 16.6 & 7.6 & 13.4 \\
Suberic acid & 16.6 & 7.3 & 13 \\
Erythritol & 17.2 & 12.6 & 35.5 \\
Xylitol & 17.5 & 14.4 & 42.5 \\
Sorbitol & 17.8 & 16.2 & 49.5 \\
$\mathrm{Et}_{4} \mathrm{NBr}$ & 15.5 & 2.8 & 3.3 \\
$\mathrm{Pr}_{4} \mathrm{NBr}$ & 15.3 & 1.6 & 1.6 \\
$\mathrm{Bu}_{4} \mathrm{NBr}$ & 15.2 & 0.4 & -0.04 \\
$\mathrm{Pe}_{4} \mathrm{NBr}$ & 15.1 & -0.8 & -1.7 \\
$\mathrm{He}_{4} \mathrm{NBr}$ & 15 & -2 & -3.3 \\
\hline
\end{tabular}


Table S5: DFT computed properties of the components. The ground state energies $E$ were determined for the components individually, in the presence of another similar molecule, in the presence of $\mathrm{H}_{2} \mathrm{O}$, and in the presence of formic acid (FA). The differences in these energies are used as characteristic properties.

\begin{tabular}{cccccc}
\hline Component & $\Delta E_{2 i}^{i}[\mathrm{eV}]$ & $\Delta E_{\mathrm{H}_{2} \mathrm{O}}^{i}[\mathrm{eV}]$ & $\Delta E_{\mathrm{FA}}^{i}[\mathrm{eV}]$ & $\Delta E_{2 i}^{\mathrm{H}_{2} \mathrm{O}}[\mathrm{eV}]$ & $\Delta E_{2 i}^{\mathrm{FA}}[\mathrm{eV}]$ \\
\hline Succinic acid & -0.805 & -0.502 & -0.239 & -0.303 & -0.566 \\
Glutaric acid & -0.771 & -0.484 & -0.397 & -0.287 & -0.374 \\
Adipic acid & -1.208 & -0.469 & -0.391 & -0.740 & -0.817 \\
Pimelic acid & -0.835 & -0.557 & -0.400 & -0.278 & -0.436 \\
Suberic acid & -0.861 & -0.716 & -0.393 & -0.144 & -0.467 \\
Erythritol & -0.414 & -0.465 & -0.394 & 0.051 & -0.020 \\
Xylitol & -0.446 & -0.284 & -0.326 & -0.161 & -0.120 \\
Sorbitol & -0.213 & -0.264 & -0.272 & 0.052 & 0.059 \\
$\mathrm{Et}_{4} \mathrm{NBr}$ & -0.826 & -0.412 & -0.498 & -0.414 & -0.328 \\
$\mathrm{Pr}_{4} \mathrm{NBr}$ & -0.374 & -0.426 & -0.486 & 0.052 & 0.112 \\
$\mathrm{Bu}_{4} \mathrm{NBr}$ & -0.204 & -0.426 & -0.496 & 0.223 & 0.292 \\
$\mathrm{Pe}_{4} \mathrm{NBr}$ & -0.174 & -0.424 & -0.493 & 0.249 & 0.319 \\
$\mathrm{He}_{4} \mathrm{NBr}$ & -0.069 & -0.405 & -0.497 & 0.336 & 0.428 \\
\hline
\end{tabular}

Table S6: Computed optimized distances of the components from DFT. The distance between the oxygen and the donating proton was determined for the components individually, in the presence of another similar molecule, in the presence of $\mathrm{H}_{2} \mathrm{O}$, and in the presence of formic acid. For the acceptors, see table S7, the optimized distance between the cation $\mathrm{N}^{+}$ and the anion $\mathrm{Br}^{-}$was determined under the same circumstances.

\begin{tabular}{ccccc}
\hline Component & $h_{i}[\AA]$ & $h_{i}^{i}[\AA]$ & $h_{i}^{\mathrm{H}_{2} \mathrm{O}}[\AA]$ & $h_{i}^{\mathrm{FA}}[\AA]$ \\
\hline Succinic acid & 0.981 & 1.010 & 0.984 & 0.990 \\
Glutaric acid & 0.981 & 1.008 & 0.983 & 0.994 \\
Adipic acid & 0.981 & 1.007 & 0.983 & 0.994 \\
Pimelic acid & 0.982 & 1.011 & 0.982 & 0.994 \\
Suberic acid & 0.982 & 1.013 & 0.987 & 0.983 \\
Erythritol & 0.974 & 0.981 & 0.980 & 0.985 \\
Xylitol & 0.975 & 0.978 & 0.978 & 0.982 \\
Sorbitol & 0.979 & 0.978 & 0.980 & 0.984 \\
\hline
\end{tabular}

Table S7: As table S6, but for the acceptors.

\begin{tabular}{ccccccc}
\hline Component & $b_{i}[\AA]$ & $b_{i}^{i}[\AA]$ & $b_{i}^{\mathrm{H}_{2} \mathrm{O}}[\AA]$ & $b_{i}^{\mathrm{FA}}[\AA]$ & $b_{\mathrm{H}_{2} \mathrm{O}}^{i}[\AA]$ & $b_{\mathrm{FA}}^{i}[\AA]$ \\
\hline $\mathrm{Et}_{4} \mathrm{NBr}$ & 4.027 & 4.266 & 4.078 & 4.069 & 0.982 & 1.020 \\
$\mathrm{Pr}_{4} \mathrm{NBr}$ & 4.037 & 4.137 & 4.119 & 4.064 & 0.982 & 1.019 \\
$\mathrm{Bu}_{4} \mathrm{NBr}$ & 4.084 & 4.122 & 4.154 & 4.100 & 0.982 & 1.019 \\
$\mathrm{Pe}_{4} \mathrm{NBr}$ & 4.174 & 4.186 & 4.273 & 4.182 & 0.981 & 1.018 \\
$\mathrm{He}_{4} \mathrm{NBr}$ & 4.188 & 4.181 & 4.236 & 4.210 & 0.982 & 1.018 \\
\hline
\end{tabular}


Table S8: Listed scores of the first six principal components of the variables.

\begin{tabular}{|c|c|c|c|c|c|c|c|}
\hline Donor & Acceptor & PC1 & $\mathrm{PC} 2$ & PC3 & $\mathrm{PC} 4$ & PC5 & PC6 \\
\hline Succinic acid & $\mathrm{Et}_{4} \mathrm{NBr}$ & 5.754 & 2.262 & 3.901 & 2.167 & 0.626 & 1.724 \\
\hline Succinic acid & $\mathrm{Pr}_{4} \mathrm{NBr}$ & 2.433 & 2.262 & 3.901 & -1.633 & -0.488 & 1.724 \\
\hline Succinic acid & $\mathrm{Bu}_{4} \mathrm{NBr}$ & 0.114 & 2.262 & 3.901 & -2.598 & -0.412 & 1.724 \\
\hline Succinic acid & $\mathrm{Pe}_{4} \mathrm{NBr}$ & -3.896 & 2.262 & 3.901 & 1.752 & -2.297 & 1.724 \\
\hline Succinic acid & $\mathrm{He}_{4} \mathrm{NBr}$ & -4.404 & 2.262 & 3.901 & 0.312 & 2.570 & 1.724 \\
\hline Glutaric acid & $\mathrm{Et}_{4} \mathrm{NBr}$ & 5.754 & 2.137 & -0.518 & 2.167 & 0.626 & 1.712 \\
\hline Glutaric acid & $\mathrm{Pr}_{4} \mathrm{NBr}$ & 2.433 & 2.137 & -0.518 & -1.633 & -0.488 & 1.712 \\
\hline Glutaric acid & $\mathrm{Bu}_{4} \mathrm{NBr}$ & 0.114 & 2.137 & -0.518 & -2.598 & -0.412 & 1.712 \\
\hline Glutaric acid & $\mathrm{Pe}_{4} \mathrm{NBr}$ & -3.896 & 2.137 & -0.518 & 1.752 & -2.297 & 1.712 \\
\hline Glutari & $\mathrm{He}_{4} \mathrm{NBr}$ & -4.404 & 2.137 & -0.518 & 0.312 & 2.570 & 1.712 \\
\hline Adipic acid & $\mathrm{Et}_{4} \mathrm{NBr}$ & 5.754 & 3.678 & 0.651 & 2.167 & 0.626 & -1.033 \\
\hline Adipic acid & $\mathrm{Pr}_{4} \mathrm{NBr}$ & 2.433 & 3.678 & 0.6 & -1.633 & -0.488 & -1.033 \\
\hline Adipic & $\mathrm{Bu}_{4} \mathrm{NBr}$ & 0.114 & 3.678 & 0.651 & -2.598 & -0.412 & -1.033 \\
\hline Adipic acid & $\mathrm{Pe}_{4} \mathrm{NBr}$ & -3.896 & 3.678 & 0.651 & 1.752 & -2.297 & -1.033 \\
\hline Adipic acid & $\mathrm{He}_{4} \mathrm{NBr}$ & -4.404 & 3.678 & 0.651 & 0.312 & 2.570 & -1.033 \\
\hline Pimelic acid & $\mathrm{Et}_{4} \mathrm{NBr}$ & 5.754 & 2.852 & -1.772 & 2.167 & 0.626 & 0.096 \\
\hline Pimelic & $\mathrm{Pr}_{4} \mathrm{NBr}$ & 2.433 & 2.852 & -1.772 & -1.633 & -0.488 & 0.096 \\
\hline Pime & $\mathrm{Bu}_{4} \mathrm{NBr}$ & 0.114 & 2.852 & -1.772 & -2.598 & 12 & 0.096 \\
\hline Pimelic acid & $\mathrm{Pe}_{4} \mathrm{NBr}$ & -3.896 & 2.852 & -1.772 & 1.752 & -2.297 & 0.096 \\
\hline Pimelic & $\mathrm{He}_{4} \mathrm{NBr}$ & -4.404 & 2.852 & -1.772 & 0.312 & 2.570 & 0.096 \\
\hline Suberic acid & $\mathrm{Et}_{4} \mathrm{NBr}$ & 5.754 & 3.488 & -2.556 & 2.167 & 0.626 & -1.413 \\
\hline Suberic & $\mathrm{Pr}_{4} \mathrm{NBr}$ & 2.433 & 3.488 & -2.556 & -1.633 & -0.488 & -1.413 \\
\hline & & 4 & 38 & -2 & -2.598 & -0.412 & -1.413 \\
\hline Suberic acid & $\mathrm{Pe}_{4} \mathrm{NBr}$ & -3.896 & 3.488 & -2.556 & 1.752 & -2.297 & -1.413 \\
\hline Suber & $\mathrm{He}_{4} \mathrm{NBr}$ & -4.404 & 3.488 & -2.556 & 0.312 & 70 & -1.413 \\
\hline Erythritol & $\mathrm{Et}_{4} \mathrm{NBr}$ & 5.754 & -3.296 & 2.153 & 2.167 & 0.626 & -1.897 \\
\hline Eryt & $\mathrm{Pr}_{4} \mathrm{NBr}$ & 2.433 & -3.296 & 2.153 & -1.633 & -0.488 & -1.897 \\
\hline & & 0.114 & -3.296 & 2.153 & -2.598 & -0.412 & -1.897 \\
\hline Eryth & $\mathrm{Pe}_{4} \mathrm{NBr}$ & -3.896 & -3.296 & 2.153 & 1.752 & -2.297 & -1.897 \\
\hline Eryt & $\mathrm{He}_{4} \mathrm{NBr}$ & -4.404 & -3.296 & 2.153 & 0.312 & 2.570 & -1.897 \\
\hline & $\mathrm{Et}_{4} \mathrm{NBr}$ & 5.754 & -5.294 & 0.250 & 2.167 & 0.626 & -1.195 \\
\hline & $\mathrm{Pr}_{4} \mathrm{NBr}$ & 2.433 & -5.294 & 0.250 & -1.633 & -0.488 & -1.195 \\
\hline & $\mathrm{Bu}_{4} \mathrm{NBr}$ & 0.114 & -5.294 & 0.250 & -2.598 & -0.412 & -1.195 \\
\hline & $\mathrm{Pe}_{4} \mathrm{NBr}$ & -3.896 & -5.294 & 0.250 & 1.752 & -2.297 & -1.195 \\
\hline $\mathrm{Xyl}$ & $\mathrm{He}_{4} \mathrm{NBr}$ & -4.404 & -5.294 & 0.250 & 0.312 & 2.570 & -1.195 \\
\hline Sorbitol & $\mathrm{Et}_{4} \mathrm{NBr}$ & 5.754 & -5.826 & -2.107 & 2.167 & 0.626 & 2.006 \\
\hline Sorbitol & $\mathrm{Pr}_{4} \mathrm{NBr}$ & 2.433 & -5.826 & -2.107 & -1.633 & -0.488 & 2.006 \\
\hline Sorbitol & $\mathrm{Bu}_{4} \mathrm{NBr}$ & 0.114 & -5.826 & -2.107 & -2.598 & -0.412 & 2.006 \\
\hline & $\mathrm{Pe}_{4} \mathrm{NBr}$ & -3.896 & -5.826 & -2.107 & 1.752 & -2.297 & 2.006 \\
\hline Sorbitol & $\mathrm{He}_{4} \mathrm{NBr}$ & -4.404 & -5.826 & -2.107 & 0.312 & 2.570 & 2.006 \\
\hline
\end{tabular}


Table S9: Loadings of the principal components 1 and 4 corresponding to the hydrogen bond acceptors. The length is the absolute distance of the variable to the origin when using both PC1 and PC4.

\begin{tabular}{ccrrc}
\hline$\#$ & Descriptor & PC1 & PC4 & Length \\
\hline 1 & $\Delta E_{2 i}^{\mathrm{FA}}[\mathrm{eV}]$ & 0.921 & 0.388 & 1.000 \\
2 & $\Delta E_{2 i}^{i}[\mathrm{eV}]$ & 0.918 & 0.394 & 0.999 \\
3 & $\Delta E_{2 i}^{\mathrm{H}_{2} \mathrm{O}}[\mathrm{eV}]$ & 0.911 & 0.409 & 0.998 \\
4 & $b_{i}[\AA]$ & 0.967 & -0.228 & 0.993 \\
5 & $b_{i}^{\mathrm{H}_{2} \mathrm{O}}[\AA]$ & 0.970 & -0.184 & 0.987 \\
6 & $b_{i}^{\mathrm{FA}}[\AA]$ & 0.930 & -0.308 & 0.979 \\
7 & $V_{\mathrm{m}}\left[\mathrm{cm}^{3} \cdot \mathrm{mol}^{-1}\right]$ & 0.988 & 0.011 & 0.988 \\
8 & $\#-\mathrm{CH}_{2}$ & 0.982 & 0.025 & 0.982 \\
9 & $M\left[\mathrm{~g} \cdot \mathrm{mol}^{-1}\right]$ & 0.982 & 0.025 & 0.982 \\
10 & $\rho\left[\mathrm{g} \cdot \mathrm{cm}^{-3}\right]$ & -0.959 & -0.217 & 0.983 \\
11 & $\delta \mathrm{H}$ & -0.982 & -0.025 & 0.982 \\
12 & $\delta \mathrm{D}$ & -0.982 & -0.025 & 0.982 \\
13 & $\delta \mathrm{P}$ & -0.982 & -0.025 & 0.982 \\
14 & $b_{i}^{i}[\AA]$ & -0.369 & -0.894 & 0.967 \\
15 & $T^{*}[\mathrm{~K}]$ & -0.923 & -0.169 & 0.938 \\
16 & $h_{\mathrm{FA}}^{i}[\AA]$ & -0.921 & 0.101 & 0.926 \\
17 & $\mathrm{H}_{2} \mathrm{O} \mathrm{solubility}[\mathrm{mol} \%]$ & -0.708 & 0.519 & 0.878 \\
18 & $\Delta S\left[\mathrm{~J} \cdot \mathrm{mol}^{-1}\right]$ & 0.603 & -0.496 & 0.781 \\
19 & $\Delta H\left[\mathrm{~kJ} \cdot \mathrm{mol}^{-1}\right]$ & 0.474 & -0.595 & 0.760 \\
20 & Proton affinity $[\mathrm{eV}]$ & -0.130 & -0.692 & 0.704 \\
21 & $h_{\mathrm{H}_{2} \mathrm{O}}^{i}[\AA]$ & -0.396 & 0.453 & 0.602 \\
22 & $\Delta E_{\mathrm{H}_{2} \mathrm{O}}^{i}$ & 0.144 & -0.517 & 0.536 \\
23 & $\Delta E_{\mathrm{FA}}^{i}$ & -0.064 & 0.426 & 0.430 \\
\hline & & & &
\end{tabular}


Table S10: Loadings of the principal components 2 and 3 corresponding to the hydrogen bond donors. The length is the absolute distance of the variable to the origin when using both $\mathrm{PC} 2$ and $\mathrm{PC} 3$.

\begin{tabular}{ccrrc}
\hline$\#$ & Descriptor & \multicolumn{1}{c}{ PC2 } & \multicolumn{1}{c}{ PC3 } & Length \\
\hline 1 & Dipole moment $[\mathrm{D}]$ & 0.976 & 0.1854 & 0.994 \\
2 & $\delta \mathrm{H}$ & 0.990 & 0.0648 & 0.992 \\
3 & $\delta \mathrm{P}$ & 0.988 & 0.0259 & 0.989 \\
4 & $\#-\mathrm{COH}$ & 0.977 & 0.1319 & 0.986 \\
5 & $\delta \mathrm{D}$ & 0.977 & 0.0890 & 0.982 \\
6 & $\mathrm{p} K_{\mathrm{a}}$ & 0.970 & -0.0290 & 0.971 \\
7 & $\Delta E_{2 i}^{i}[\mathrm{eV}]$ & 0.922 & 0.0809 & 0.925 \\
8 & $\Delta E_{2 i}^{\mathrm{FA}}[\mathrm{eV}]$ & 0.898 & 0.1680 & 0.914 \\
9 & $\#-\mathrm{CH}_{2}$ & -0.892 & 0.4118 & 0.982 \\
10 & $h_{i}^{i}$ & -0.981 & 0.0473 & 0.982 \\
11 & $\#=\mathrm{CO}$ & -0.976 & 0.0366 & 0.977 \\
12 & $V_{\mathrm{m}}\left[\mathrm{cm}^{3} \cdot \mathrm{mol}^{-1}\right]$ & -0.157 & 0.9082 & 0.922 \\
13 & $\rho\left[\mathrm{g}^{-1} \mathrm{~cm}^{-3}\right]$ & 0.652 & -0.6080 & 0.891 \\
14 & $M\left[\mathrm{~g} \cdot \mathrm{mol}^{-1}\right]$ & 0.144 & 0.8789 & 0.891 \\
15 & $h_{i}[\AA]$ & -0.830 & 0.2640 & 0.871 \\
16 & $h_{i}^{\mathrm{H} \mathrm{O}}$ & -0.862 & 0.1184 & 0.870 \\
17 & $\mathrm{H}_{2} \mathrm{O} \mathrm{solubility}[\mathrm{mol} \%]$ & 0.822 & 0.2598 & 0.862 \\
18 & $\Delta E_{\mathrm{H}_{2} \mathrm{O}}$ & 0.825 & -0.1290 & 0.835 \\
19 & Proton affinity $[\mathrm{eV}]$ & 0.227 & 0.7980 & 0.830 \\
20 & $T^{*}[\mathrm{~K}]$ & -0.548 & -0.6040 & 0.816 \\
21 & $\Delta H\left[\mathrm{~kJ} \cdot \mathrm{mol}^{-1}\right]$ & 0.035 & -0.7190 & 0.720 \\
22 & $\Delta E_{2 i}^{\mathrm{H}_{2} \mathrm{O}}[\mathrm{eV}]$ & 0.688 & 0.1764 & 0.710 \\
23 & $h_{i}^{\mathrm{FA}}[\AA]$ & -0.684 & -0.1350 & 0.697 \\
24 & $\Delta S\left[\mathrm{~J} \cdot \mathrm{mol}^{-1}\right]$ & 0.276 & -0.5780 & 0.641 \\
25 & $\Delta E_{\mathrm{FA}}^{i}$ & 0.396 & -0.3760 & 0.546 \\
\hline & & & &
\end{tabular}



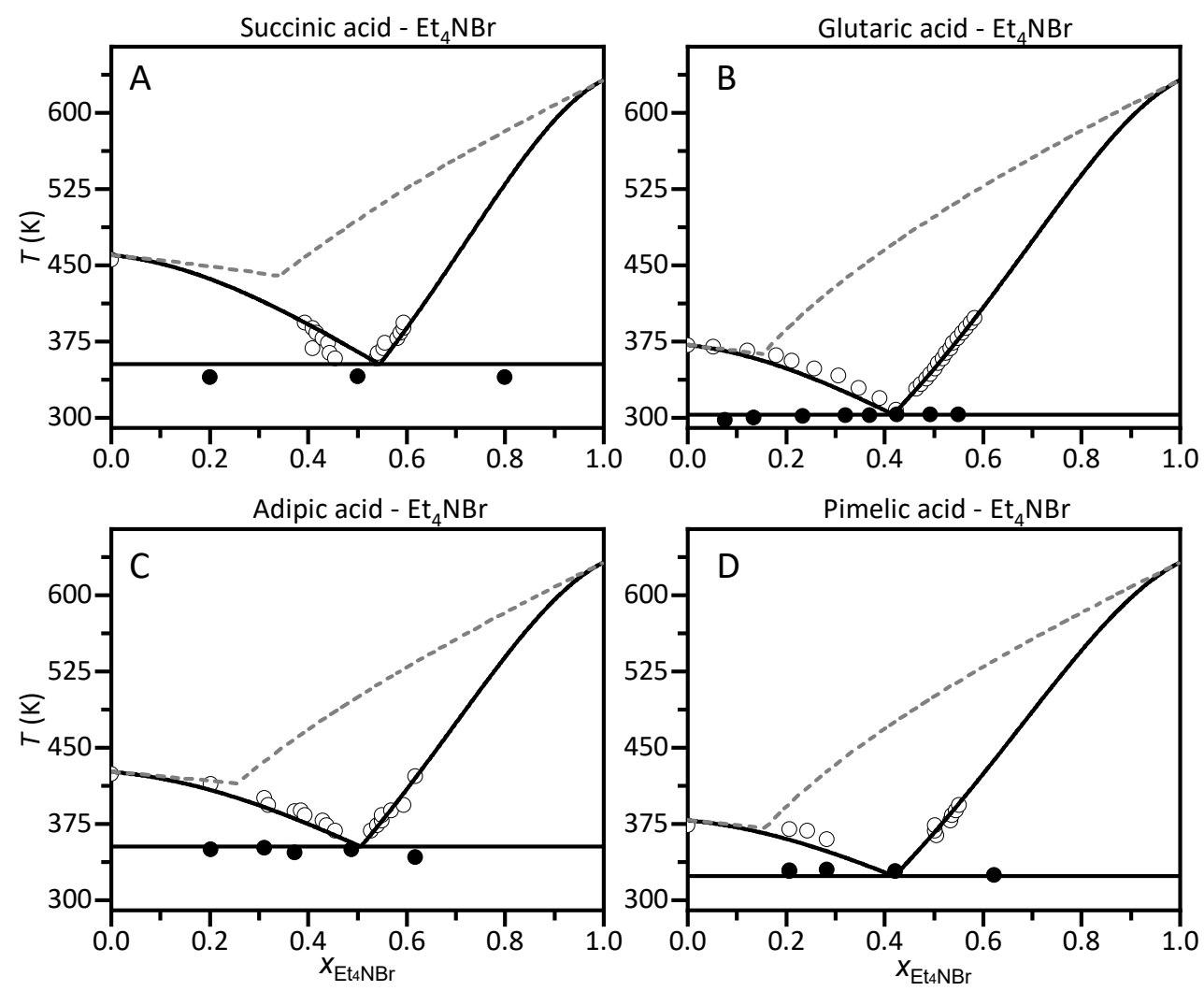

Figure S1: Phase diagrams of binary mixtures of $\mathrm{Et}_{4} \mathrm{NBr}$ with succinic acid (A), glutaric acid (B), adipic acid (C), and pimelic acid (D) used to estimate the fusion properties of $\mathrm{Et}_{4} \mathrm{NBr}$. The filled circles are solidus data points, the open circles represent the liquidus points. The grey dashed curves are the predictions of ideal behaviour, the black curves describe the phase behaviour resulting from the RS theory fit. The resulting fusion properties of $\mathrm{Et}_{4} \mathrm{NBr}$ are $T^{*}=633 \mathrm{~K}$ and $\Delta H=13.9 \mathrm{~kJ} \cdot \mathrm{mol}^{-1}$. The RS theory description yielded the following interaction parameters for the binary mixtures (A) Succinic acid, $\chi=-7.06$; (B) Glutaric acid, $\chi=-5.87$; (C) Adipic acid, $\chi=-5.81$; (D) Pimelic acid, $\chi=-5.86$. 

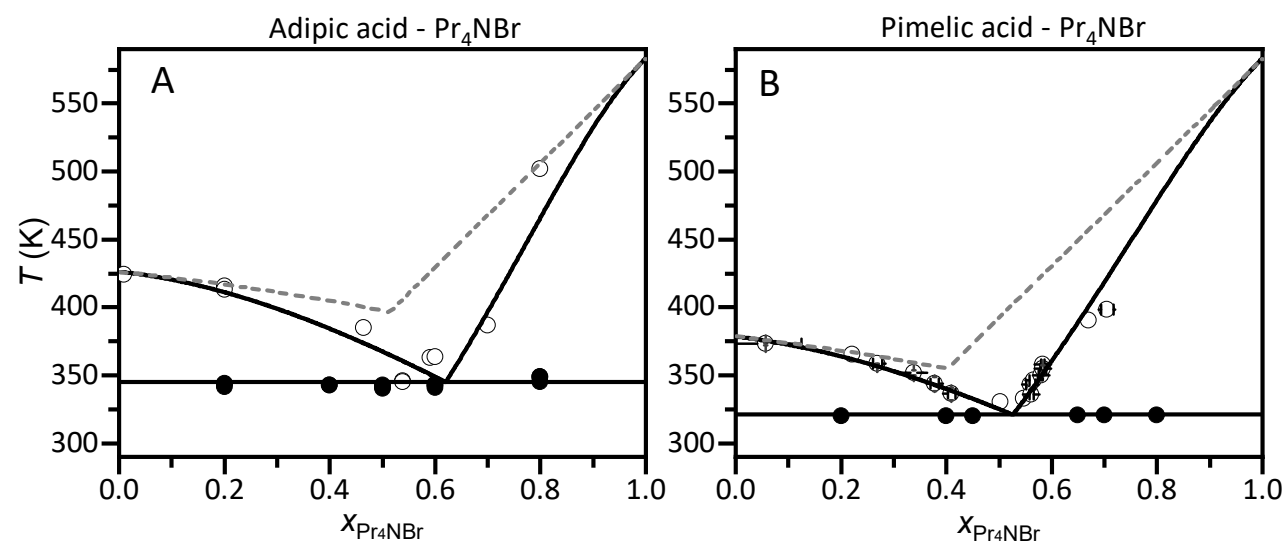

Figure S2: Phase diagrams of binary mixtures of $\mathrm{Pr}_{4} \mathrm{NBr}$ with adipic acid (A) and pimelic acid (B) used to estimate the fusion properties of $\mathrm{Pr}_{4} \mathrm{NBr}$. The filled circles are solidus data points, the half circles represent the liquidus points. The grey dashed curves are the predictions of ideal behaviour, the black curves describe the phase behaviour resulting from the RS theory fit. The resulting fusion properties of $\operatorname{Pr}_{4} \mathrm{NBr}$ are $T^{*}=583 \mathrm{~K}$ and $\Delta H=7.3$ $\mathrm{kJ} \cdot \mathrm{mol}^{-1}$. The RS theory description yielded the following interaction parameters for the binary mixtures: (A) Adipic acid, $\chi=-3.71$; (B) Pimelic acid, $\chi=-2.46$. 

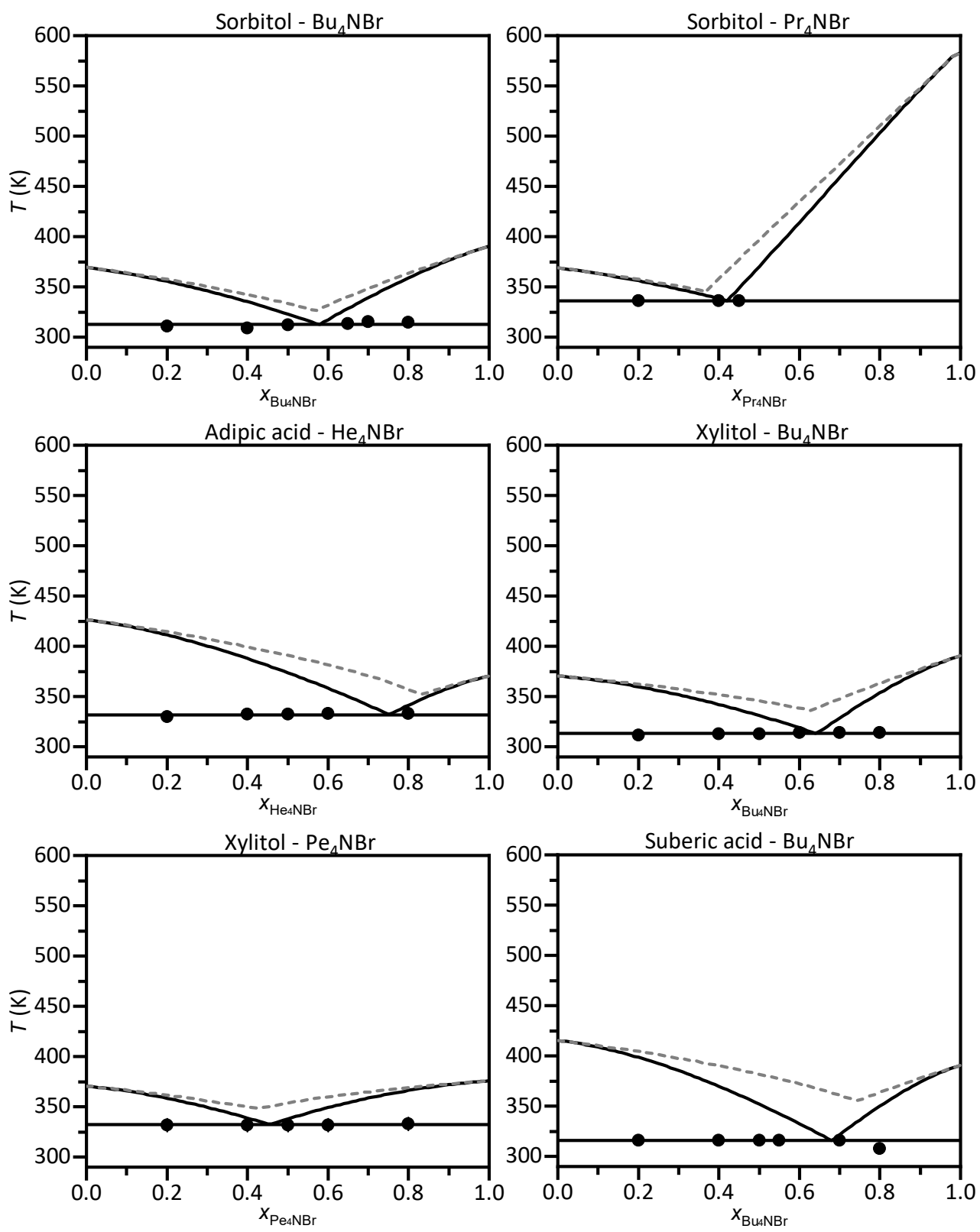

Figure S3: Predicted phase diagrams of selected binary mixtures of mentioned components and measured eutectic temperatures. The predicted ideal phase boundaries, thus without enthalpic interactions are dashed. The solubility curves predicted by regular solution theory with the interaction parameter $\chi$ obtained using the measured eutectic temperature (black data points) are solid. 

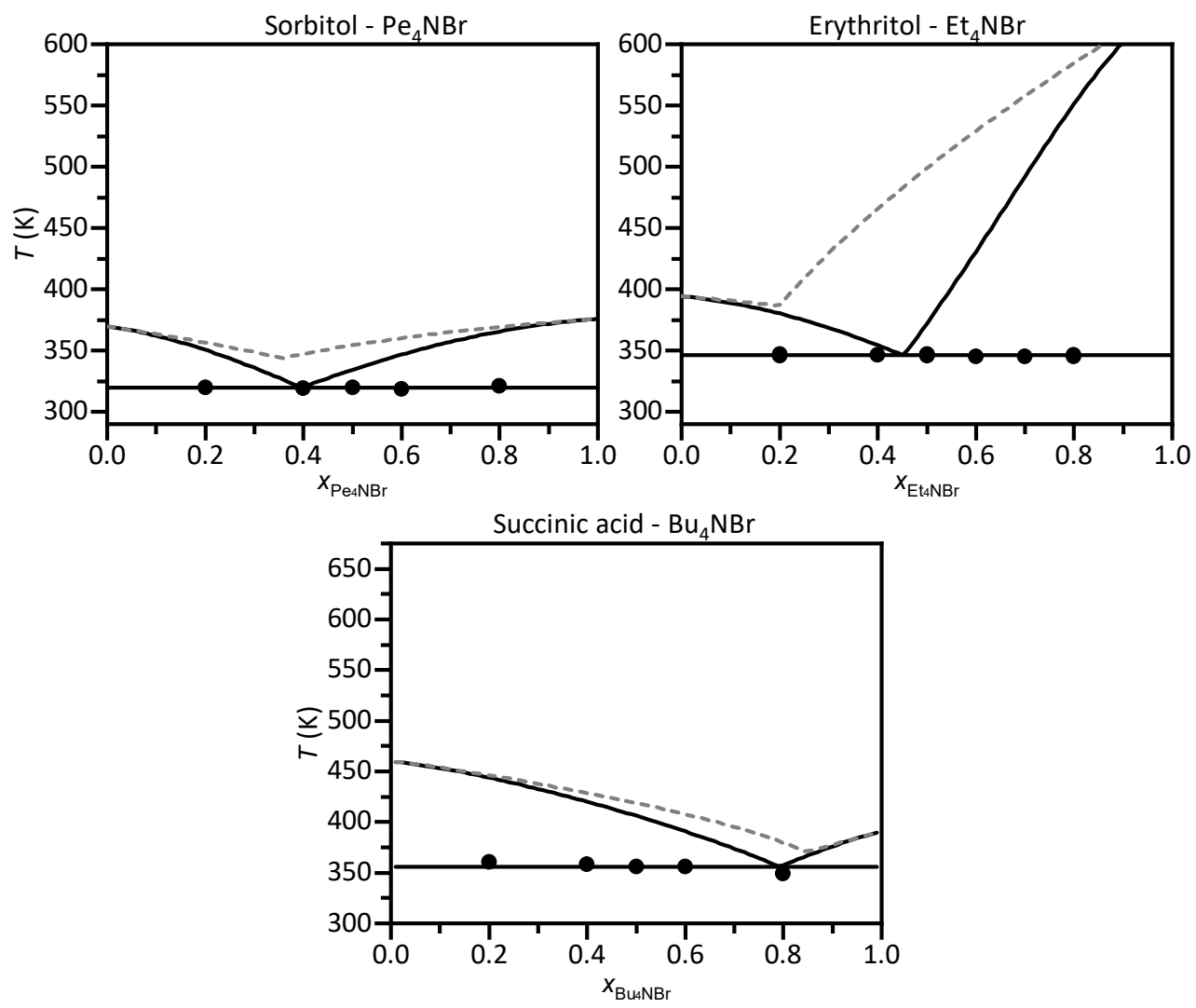

Figure S3: Continued. 


\section{References}

(1) GESTIS-Stoffdatenbank. December 2018; https://www.dguv.de/ifa/gestis/ gestis-stoffdatenbank/index.jsp.

(2) Lide, D. R., Ed. Handb. Chem. Phys., 76th ed.; CRC press: Boca Raton, 1995; Chapter 3 , pp 3-152.

(3) Bretti, C.; Crea, F.; Foti, C.; Sammartano, S. Solubility and activity coefficients of acidic and basic nonelectrolytes in aqueous salt solutions. J. Chem. Eng. Data 2006, $51,1660-1667$.

(4) Bouchard, A.; Hofland, G. W.; Witkamp, G.-J. Properties of sugar, polyol, and polysaccharide water-ethanol solutions. J. Chem. Eng. Data 2007, 52, 1838-1842.

(5) Wang, S.; Li, Q. S.; Su, M. G. Measurement and correlation of solubility of xylitol in binary ethanol + acetone solvent mixtures with the combined nearly ideal binary solvent/ Redlich - Kister equation. J. Chem. Eng. Data 2007, 52, 1733-1735.

(6) Peddle, C.; Turner, W. Solubilities of salts of ammonium bases in water and in chloroform. Part I. Solubility as alpha-constitutive property. J. Chem. Soc. 1913, 103, 12021209.

(7) Nakayama, H. Solid-liquid and liquid-liquid phase equilibria in the symmetrical tetraalkylammonium halide-water systems. Bull. Chem. Soc. Jpn. 1981, 54, 3717-3722.

(8) Lindvig, T.; Michelsen, M. L.; Kontogeorgis, G. M. A Flory-Huggins model based on the Hansen solubility parameters. Fluid Phase Equilib. 2002, 203, 247-260.

(9) Stefanis, E.; Panayiotou, C. Prediction of Hansen solubility parameters with a new group-contribution method. Int. J. Thermophys. 2008, 29, 568-585. 